\title{
Choosing the Packet Size in Multi-hop Underwater Networks
}

\author{
Stefano Basagni*, Chiara Petrioli ${ }^{\dagger}$, Roberto Petroccia ${ }^{\dagger}$ and Milica Stojanovic* \\ * ECE Department \\ Northeastern University \\ \{basagni, millitsa\}@ece.neu.edu \\ $\dagger$ Dipartimento di Informatica \\ Università di Roma "La Sapienza" \\ $\{$ petrioli, petroccia\}@di.uniroma1.it
}

\begin{abstract}
Two MAC layer protocols are considered for multi-hop underwater acoustic networks: Pure CSMA, suitably configured to perform over a long-delay channel, and the Distance-Aware Collision Avoidance Protocol (DACAP), a protocol specifically designed for collision avoidance via a distributed coordination function à la IEEE 802.11. We investigate the impact of packet size on the performance of these two protocols. A comparative analysis, conducted via ns- 2 simulations, quantifies throughput efficiency, end-to-end delay and energy-per-bit consumption as functions of the packet size. The results clearly indicate the existence of an optimal packet size for each scenario. The optimal packet size depends on the protocol characteristics, on the offered load, and is heavily influenced by the bit error rate. The results also reveal performance sensitivity to the choice of the packet size for the different protocols (CSMA and DACAP), emphasizing how a wrong selection of the packet size can result in a higher cost to performance.
\end{abstract}

\section{INTRODUCTION}

Current solutions for acoustic networking, which mostly concern single-hop topologies, are summarized in surveys [1], [2]. More recently, the emphasis has shifted toward multi-hop networking as a means to provide wider area coverage with increased efficiency [3]. Here, we focus on underwater multi-hop scenarios, and address the issue of packet length selection for maximizing the throughput efficiency, defined as the ratio between the effective (delivered) and the offered (attempted) bit rate.

The design of underwater MAC and routing protocols has flourished in the past few years [4]-[14]; however, only a few analyses are concerned with parameter optimization, and in particular with the packet size selection for a given transmission range, bit rate, and the error probability. Stojanovic [15] has investigated packet length optimization for maximizing throughput efficiency at the data link layer (point-to-point scenario). That work, aimed at providing measures to counteract the limitations of "Stop and Wait" (S\&W) MAC protocols in half-duplex acoustic channels, shows that their performance depends heavily on the choice of the packet size. In adapting the terrestrial Multiple Access Collision Avoidance (MACA) protocol [16] to underwater acoustics, $\mathrm{Ng}$ et al. [17] define MACA-U as a MAC protocol for underwater multi-hop networks. Although investigating only three different packet sizes (150B, 300B and 600B), that paper clearly shows the remarkable impact that the packet size has on throughput. In particular, since MACA is based on a Request-To-Send/Clear-To-Send (RTS/CTS) handshake, it is shown that, at least for the considered size range, and given a grid nodal deployment and an ideal channel $(B E R=0)$, the longer the packet, the higher the throughput.

In this paper we consider two realistically deployable underwater MAC protocols, namely, pure Carrier Sense Multiple Access (CSMA) and the Distance-Aware Collision Avoidance Protocol (DACAP) [8], which exemplify MAC schemes with and without RTS/CTS handshake, respectively. Our ns-2-based simulations consider several different characteristics of underwater systems, including realistic bit rates, energy consumption models, and different bit error rates (BERs). Results show that the packet size drastically affects network performance; in other words, that a wrong selection can result in a higher performance penalty. As preliminarily shown in [15] for throughput in single-hop communications, crucial metrics such as throughput efficiency, latency, and energy consumption in multi-hop underwater networks can be greatly improved by a judicious choice of the packet size. The best packet size depends on the data generation rate, the channel bit rate and the BER. We also show that there are packet sizes that detrimentally affect network performance independently of those parameters: These sizes should not be used with the channel access methods investigated here.

The remainder of paper is organized as follows. Section II describes the details of CSMA and DACAP. Their performance is evaluated in Section III. Concluding remarks are given in Section IV. 


\section{CSMA AND DACAP}

We describe here the two protocols considered in our study. The reader who is familiar with these protocols may wish to skip to Section III.

1. CSMA (Carrier Sensing Multiple Access) is a wellknown protocol for channel access [16]. When a node has a data packet to transmit, it first checks whether the channel is idle or busy. In the first case, it starts the packet transmission. If the channel is busy, the node delays the transmission according to the CSMA exponential backoff mechanism. We consider two versions of this protocol. The first is the following: A node that transmits a data packet receives no feedback about whether the intended recipient has received it or not. The second adds robustness by having the destination node acknowledge the data reception to its source. If the ACK is not received within a given time (set to 2Delay + ackTime), the data packet is re-transmitted either till successful reception, every time choosing the backoff time in an interval twice as long as the previous one, or till the maximum limit of retries has been reached. Here, Delay is the transmission delay between source and destination. Its value is initially set to maxDelay and successively set by the nodes to a value computed according to the (estimated) distance between source and destination (which is based on the time difference between data packet transmission and ACK reception). The backoff time is chosen randomly and uniformly in $[0, T]$, where $T=2^{\text {txRetry }}$ (2maxDelay). Retransmission of the same packet stops after a predefined number of times.

2. DACAP (Distance Aware Collision Avoidance Protocol [8]) uses the RTS/ CTS handshake for reserving the channel for packet transmission. More specifically, when a node has a data packet to send, it checks the channel, and if the channel is idle it transmits an RTS. Upon correctly receiving it, the destination replies right away with a CTS. It then waits for the data packet. With respect to the usual CSMA/CA scheme, DACAP adapts to the underwater channel characteristics by using the following mechanism. If while waiting for a data packet, a destination node overhears a control packet for some other node, it sends a very short WARNING packet to its sender. Upon receiving a CTS, a sender waits for some time, $T_{\text {warning, }}$, before transmitting the data packet. If it overhears another control packet or receives a WARNING packet from the destination during this time, the sender node aborts transmission. The length of the WARNING time depends on the distance between the source and destination, which the sender can learn by measuring the RTS/CTS round-trip time. When the receiver overhears an RTS and sends a warning, it does not know whether the warning will reach the sender on time to make it abort the transmission. Since a data packet can still arrive, the receiver must continue listening to the channel even after having sent a warning. For this reason, the WARNING time is defined as the minimum waiting period between receiving the CTS and sending the data that guarantees absence of harmful collisions. Potential interferers are blocked as usually in RTS/CTS schemes.

\section{Performance Evaluation}

CSMA and DACAP have been implemented in the VINT project ns-2 simulator [18] extended to include key characteristics of the underwater environment such as 3D nodal deployment, propagation at the speed of sound, frequency-dependent acoustic path loss, and spatially-varying interference of all the active nodes that may result in packet losses [19].

\section{A. Simulation scenarios and settings}

We consider a scenario where 100 static nodes are placed on the seafloor at a depth of $200 \mathrm{~m}$. The nodes are randomly and uniformly scattered within a $4 \mathrm{~km} \times 4 \mathrm{~km}$ footprint. Packets are transmitted from the nodes to a common sink (data collection point) located centrally on the surface.

Nodes are equipped with an acoustic modem with transmission range $R=1000 \mathrm{~m}$. Communication occurs in a multi-hop fashion over pre-determined shortest path routes.

Each packet that makes it to the sink traverses an average of 2.3 hops (the maximal number of hops is 4 ). The transmitting power is computed according to [19]. It is adjusted to achieve a SNR of $20 \mathrm{~dB}$ at a receiver that is $1000 \mathrm{~m}$ away, taking into account the ambient noise power, and the frequency-dependent acoustic path loss. Receiving power, idle and transmission powers are set to $100 \mathrm{~mW}, 100 \mathrm{~mW}$ and to $4930 \mathrm{~mW}$, respectively.

The carrier frequency is $24 \mathrm{KHz}$. This frequency also defines the interference radius, i.e., the inter-node distance within which transmissions can interfere. In general, this distance will be greater than the transmission radius.

We consider different data packet payloads and data rates to assess the protocol performance with respect to these values. Data packet payloads range from 50B to 3000B, in increments of 50B (for a total of 60 different packet sizes). We consider acoustic modems with two possible bit rates, $R_{b}=200 \mathrm{bps}$ and $2000 \mathrm{bps}$. Once fixed, the data rate is the same for all nodes. Two different bit error rates are considered, $\mathrm{BER}=10^{-4}$ and $10^{-6}$. Traffic is generated according to a Poisson process with aggregate (network-wide) rate of $\lambda$ packets per second. We also define the normalized packet rate as $\bar{\lambda}=\lambda T_{\text {pack }}$, whose values are considered in the range 0 to 1 packets per packet time. The packet time can be express as $T_{\text {pack }}=N_{b} / R_{b}$, where $N_{b}$ is the packet 
size in bits. The simulations presented here concern very low traffic $(\bar{\lambda}=0.01)$, low traffic $(\bar{\lambda}=0.1)$, medium traffic $(\bar{\lambda}=0.3)$ and high traffic $(\bar{\lambda}=0.6)$. Results from simulations with very low traffic are shown only for scenarios where the nodes transmit at 2000bps, since at this speed nodes inject a greater number of packets into the channel. Results for the high traffic load are shown only for scenarios where nodes transmit at lower bit rates. Once a packet is generated it is associated with a source selected randomly among all the nodes. The destination of all the packets is the sink.

The total size of a data packet is given by the payload plus the headers added by the different layers (physical through network). The physical layer header contains all the information needed by the modem to correctly start receiving a packet (synchronization preamble, delimiters, etc.) At the physical layer, nodes need a synchronization peering time which is taken to be on the order of $10 \mathrm{~ms}$ (the physical header overhead changes according to the data rate). The MAC header contains the sender's and the destination's IDs, and the packet type. The MAC header length is set to $3 \mathrm{~B}$. The size of RTS and CTS packets are set to $6 \mathrm{~B}$, and $\mathrm{ACK}$ and WARNING packets are $3 \mathrm{~B}$ long. To correctly receive each packet (control or data) the signal to interference ratio at the receiver is required to be $\mathrm{SIR} \geq 15 \mathrm{~dB}$. Each node has a buffer of $30 \mathrm{~KB}$ where data coming from the upper layers are stored before transmission. Whenever the buffer is full and a new packet arrives, the oldest packet is discarded. We also limit the number of packets that can be stored to 50 . In this way (especially for smaller packet sizes) nodes are not filling their buffers with old information. Our implementation of CSMA mandates to discard a packet after 7 failed transmission attempts, or 7 failed attempts to access the channel. The same holds for DACAP concerning RTS packets: After 7 attempts to access the channel, or after 7 failed retransmissions, a data packet is discarded. Every point reported in Figures 1 to 9 has been obtained by averaging over the number of trials needed to achieve a statistical confidence of $95 \%$ with a $5 \%$ precision.

\section{B. Simulation}

To capture the effectiveness and costs of delivering bits to the sink, the following metrics are used.

(a) Throughput Efficiency, defined as the ratio between the average bit rate delivered to the sink (correct bits) and the average bit rate offered by the network, $N_{b} \lambda$.

(b) End-to-end latency per meter, defined as the time between the packet generation and the time of its correct delivery at the sink, divided by the distance between source and destination. Normalization by the distance is used so as to unify the performance over a varying coverage area (a larger area will entail proportionately larger propagation delay). A protocol that keeps this metric constant for a varying coverage area can thus be considered scalable. This metric is computed only for the packets correctly delivered, and averaged over all the packets.

(c) Energy per bit, defined as the energy consumed by the network to correctly deliver a bit of data to the sink, averaged over all the nodes.

In what follows, we discuss these performance measures as functions of the packet size, considering different bit rates $R_{b}$, and different BERs.

\section{Throughput efficiency}

Figures 1 and 2 show the throughput efficiency at BER of $10^{-6}$ and $10^{-4}$, with transmission rates $200 \mathrm{bps}$ and $2000 \mathrm{bps}$. At $\mathrm{BER}=10^{-6}$, the throughput efficiency steadily increases with the packet size, reaching a maximum that depends on the offered load. Results show that "the longer the packet, the better the utilization." In contrast to this situation, at BER of $10^{-4}$, there clearly exists an optimal packet size for which the throughput efficiency is maximized. This packet size strikes the best trade-off between the time lost in waiting for ACKs and the time spent in re-transmissions. Longer packets entail a higher probability of packet error.

Focusing on the results of Figure 1(a), we see that at 200bps and low traffic, both protocols deliver over $90 \%$ of bits when the packet size is $150 \mathrm{~B}$ or more (shorter packets imply higher overhead, especially with DACAP, causing a reduction in the throughput efficiency). As the traffic increases, the throughput efficiency decreases. The maximal efficiency is on the order of $80 \%$ for medium traffic for both protocols, and on the order of $50 \%$ (CSMA) and 40\% (DACAP) for high traffic. Throughput efficiency drops with offered load because of multi-hopping, where each hop generates new overhead and extra packets (ACKs for both protocols and RTS/ CTS for DACAP). The number of collisions increases also due to interference from transmitting nodes multiple hops away, unless regulated through power control. CSMA has lower waiting time but suffers more from the re-transmissions. DACAP pays a high overhead in terms of delays accessing the channel, especially when short data packets are used, so that there is a higher number of packets is in the network, each experimenting a long waiting time. When the data payload increases DACAP suffers less from the propagation delay and starts increasing the amount of data correctly delivered to the sink.

Figure 1(b) shows the throughput efficiency when the raw transmission capability of each node is increased to 2000 bps. In such a situation transmission delays are ten times shorter while the propagation delay is the same. It is interesting to note that a higher throughput 


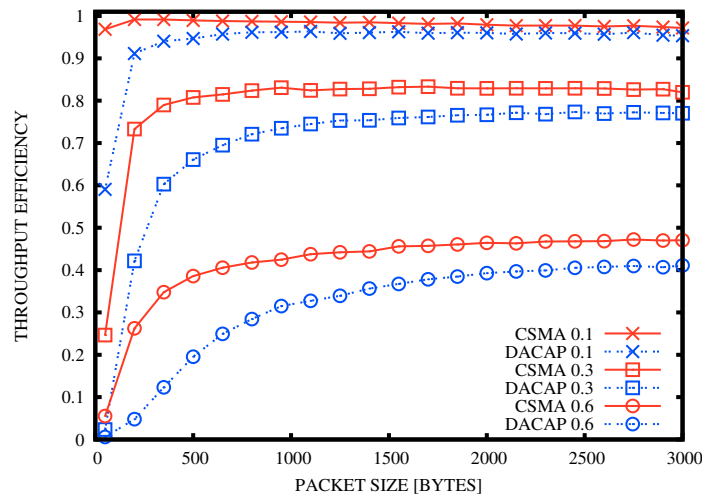

(a) $R=200 \mathrm{bps}$

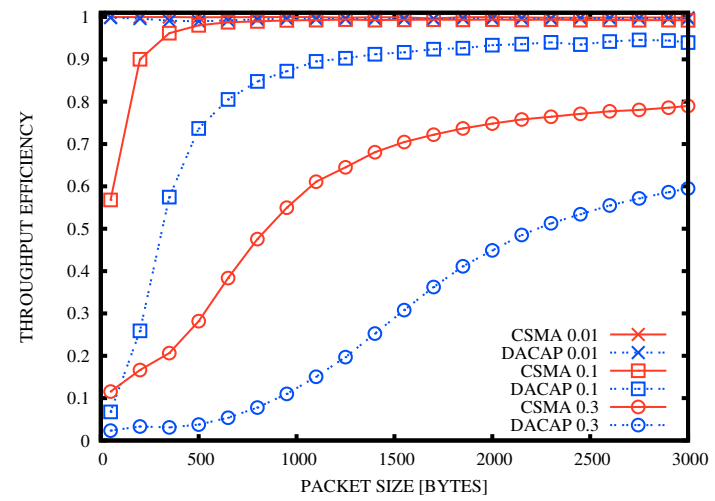

(b) $R=2000 \mathrm{bps}$

Figure 1. Throughput efficiency for $\mathrm{BER}=10^{-6}$.

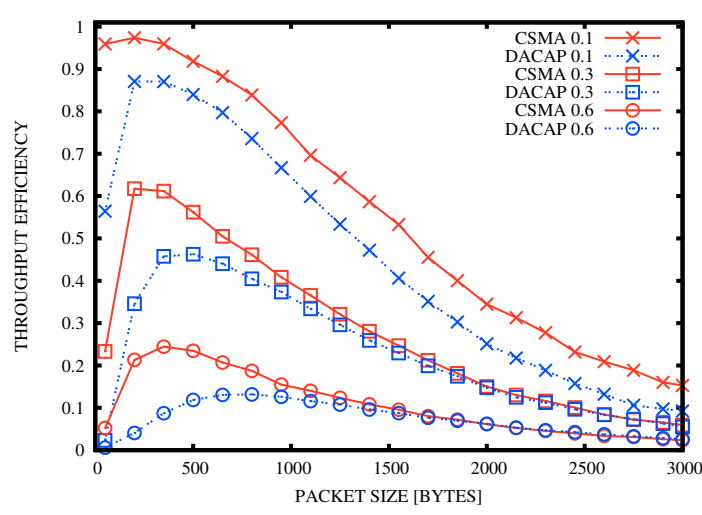

(a) $R=200 \mathrm{bps}$

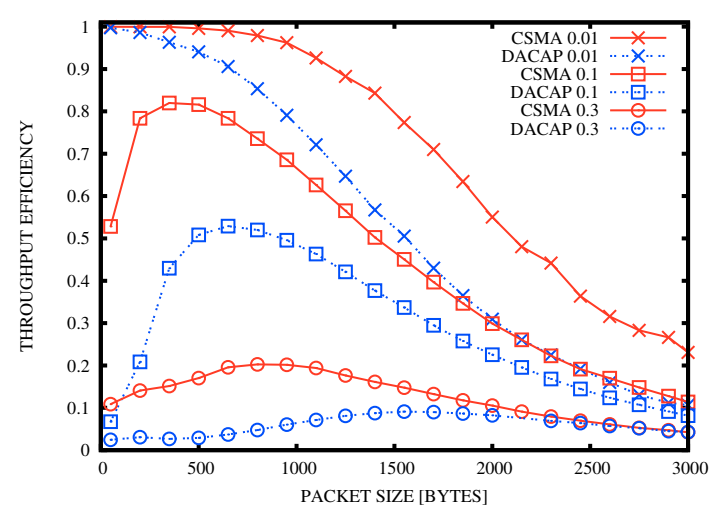

(b) $R=2000 \mathrm{bps}$

Figure 2. Throughput efficiency for $\mathrm{BER}=10^{-4}$.

efficiency is now achieved for the same effective offered load, i.e. the offered load measured in bits per second. For example, at $\bar{\lambda}=0.01$ packets per packet time both protocols achieve almost $100 \%$ efficiency, but the effective offered load is $\bar{\lambda} R=20$ bps with $R=2000$ bps and 2 bps with $R=200$. To achieve an effective offered load of $20 \mathrm{bps}$ with $R=200$ we have to consider $\bar{\lambda}=0.1$, and the efficiency there is about $90 \%$. Considering the same offered load in terms of bits per second generated in the network, the higher the data rate, the higher the throughput, because each protocol experiences ten times shorter delays, speeding up the packet delivery and reducing the probability of collisions. At the same value of packets per packet time, protocols do not reach the same throughput efficiency at a higher bit rate. This is a consequence of the fact that increasing the bit rate means increasing the number of data packets generated in the network.

As the bit error rate increases, the situation changes considerably (Figure 2). The throughput no longer increases steadily with the packet size, but instead reaches a maximum and decreases thereafter. The value of the maximum depends on the offered load, the bit rate, and the BER. The effect of increased BER is significant: for example, at $\bar{\lambda}=0.3$ and $R=200 \mathrm{bps}$, the maximal achievable throughput is about $60 \%$ for CSMA and $50 \%$ for DACAP, quite a decrease from the $80 \%$ at BER $10^{-6}$. The desired range of operation is in the stable region (to the left of maximum), i.e. with packets slightly shorter than the optimum. It is also important to note the sensitivity of throughput performance to the selection of packet size. Looking at the same example, $\bar{\lambda}=0.3$ and $R=200$ bps, where the optimal packet size is about $350 \mathrm{~B}$ for CSMA and 500B for DACAP, we note that a system design with 1500B packets would result in a throughput of only $30 \%$. This is a significant loss compared to the optimal $60 \%$ and $50 \%$, a fact that emphasizes the importance of careful packet size selection. Comparing the results of Figures 2(a) and 2(b) we see again that the overall performance is affected by the bit rate, similarly as in the case of low BER.

Figure 3 summarizes the results for throughput effi- 


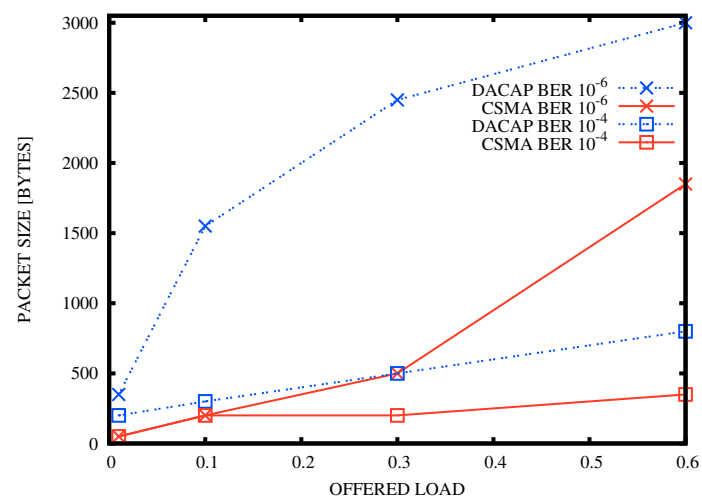

(a) $R=200 \mathrm{bps}$

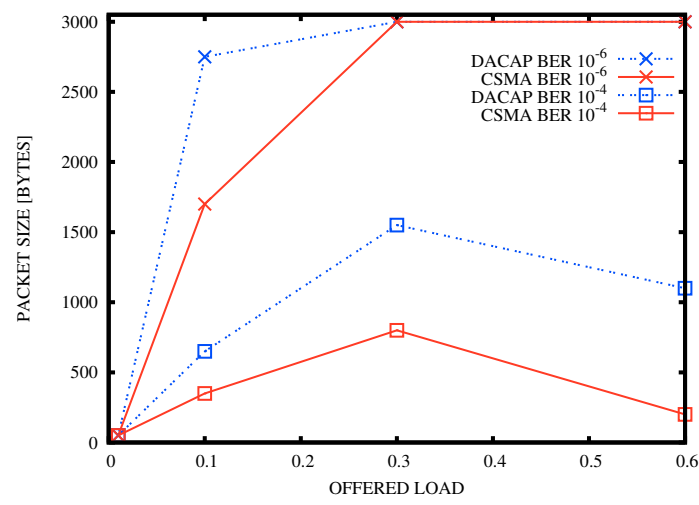

(b) $R=2000 \mathrm{bps}$

Figure 3. Packet sizes that optimize throughput efficiency.

ciency and the corresponding optimal packet size. Ties are broken based on packet latency per meter and energy consumption values. These results can readily be used as a rough guideline for the design of practical systems. It is clear how DACAP, being more affected by the propagation delay, shows the best performance with longer packet sizes. Not having to pay extra delays for accessing the channel, CSMA instead prefers short data packets when the traffic load is low. As the traffic load increases, longer packets result in a lower number of channel accesses for the same information throughput. This is why the maximum throughput efficiency is achieved at higher values of the packet size.

When the bit rate increases (Figure 3(b)) the optimal packet size is uniformly higher for DACAP than for CSMA. The throughput efficiency is similar for both protocols and latency per meter comes into play to break the tie.

As the bit error rate increases, longer packets are more affected by the probability of bit flipping. The optimal packet size thus decreases with BER.

\section{Packet latency per meter}

Packet latency per meter is chosen over the more common "absolute latency" as a more informative measure for situations in which the nodes are at different distances from the sink. Furthermore, this metric could be used to compare protocols over scenarios where the number of the nodes and the size of the deployment area vary considerably. In this case, protocols that keep the normalized delay constant can be considered more scalable than those that do not.

Figure 4 shows the average packet latency per meter when the channel BER is $10^{-6}$ in networks with nodes transmitting at the two bit rates considered. Figure 5 zooms into the more interesting region to emphasize the differences among protocols.
As expected, the lower the traffic the lower the delay. We also notice that at low bit rate (Figure 4(a) and Figure 5(a)) CSMA and DACAP incur high latency per meter at small packet sizes. As the packets size increases the normalized delay decreases, reaching a minimum and then starting to increase again. When the packet size is short, both CSMA and DACAP experience high packet latency. Having more packets in the network, CSMA is more affected by collisions and re-transmissions, while DACAP suffers more from the waiting delay. The described trends are different when the traffic is extremely low. In this case, both protocols experience similar delay performance and the delay increases with the packet size.

Increasing the bit rate significantly improves the delay performance (Figure 5(b)). At very low traffic $(\bar{\lambda}=$ 0.01 ), the propagation delay and the time needed for re-transmission due to an occasional collision are the main components of the delay. A similar observation can be made for low traffic scenarios $(\bar{\lambda}=0.1)$, with the exception of CSMA for packet sizes smaller than 500B. At higher traffic load, the normalized delays increase. Both protocols start reducing the delay as soon as the number of packets in the network decreases and there are less collisions and re-transmissions (CSMA) and less time is spent for the handshake to access the channel (DACAP).

Results for the higher BER are shown in Figure 6 and Figure 7. We can see similar performance with respect to the case of lower BER. Latencies are slightly longer because of the higher number of re-transmissions. Longer packets and higher BER imply higher probability of packet error so that the number of payload bits correctly delivered to the sink is drastically reduced. When long packets have to go through more than one link and each link has a high probability of packet error, the number of packets discarded increases with the number of links traversed. For this reason, the majority 


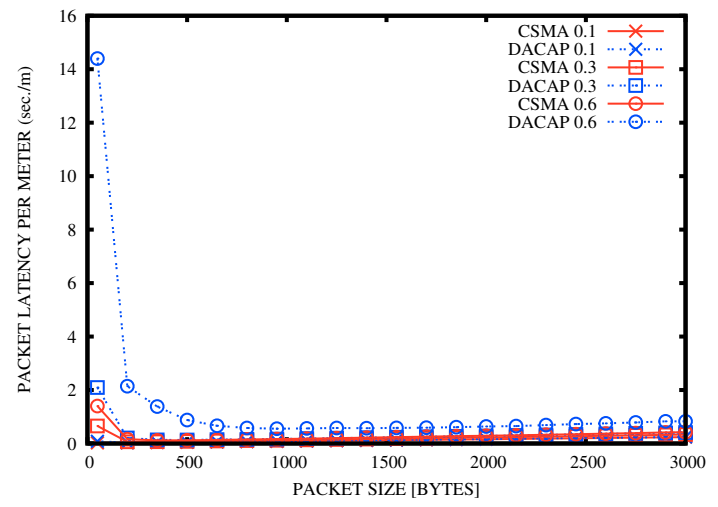

(a) $R=200 \mathrm{bps}$

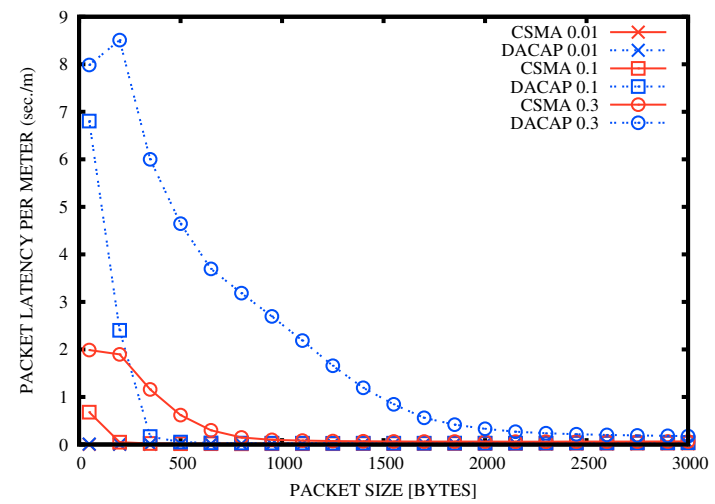

(b) $R=2000 \mathrm{bps}$

Figure 4. End-to-end latency per meter for BER $=10^{-6}$.

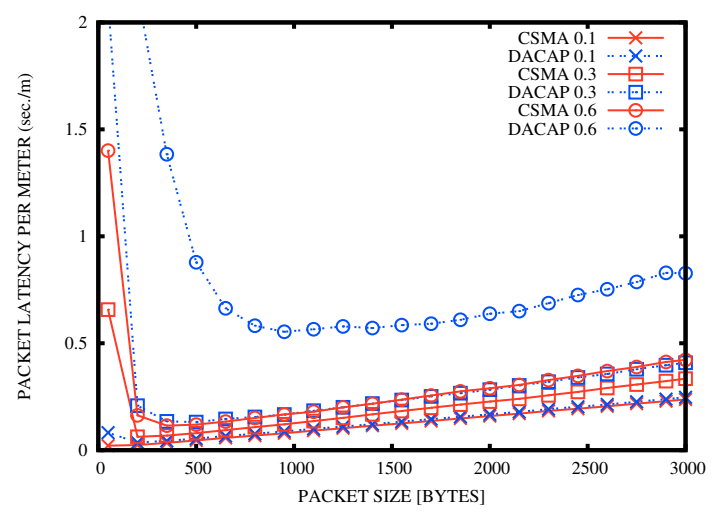

(a) $R=200 \mathrm{bps}$

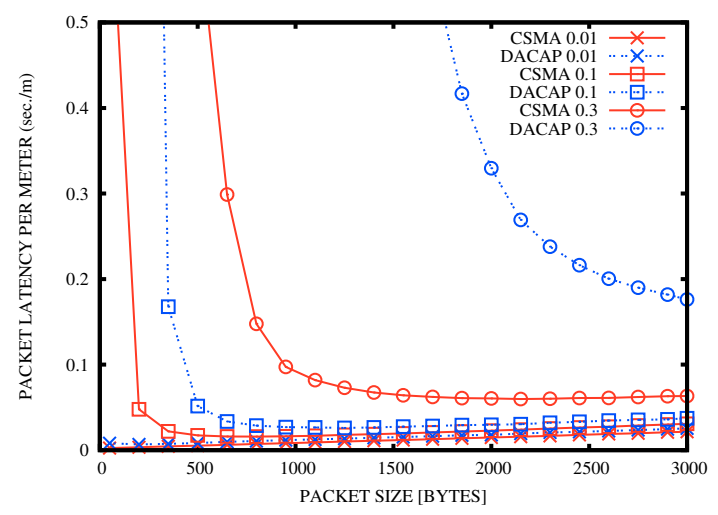

(b) $R=2000 \mathrm{bps}$

Figure 5. Zoom into the end-to-end latency per meter for $\mathrm{BER}=10^{-6}$.

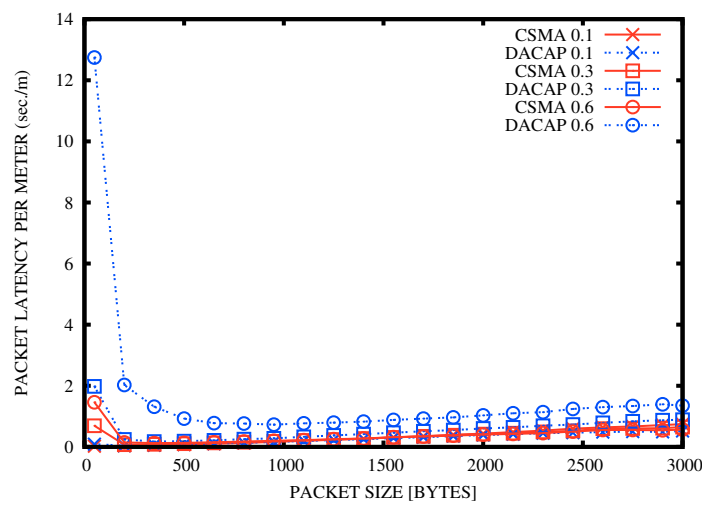

(a) $R=200 \mathrm{bps}$

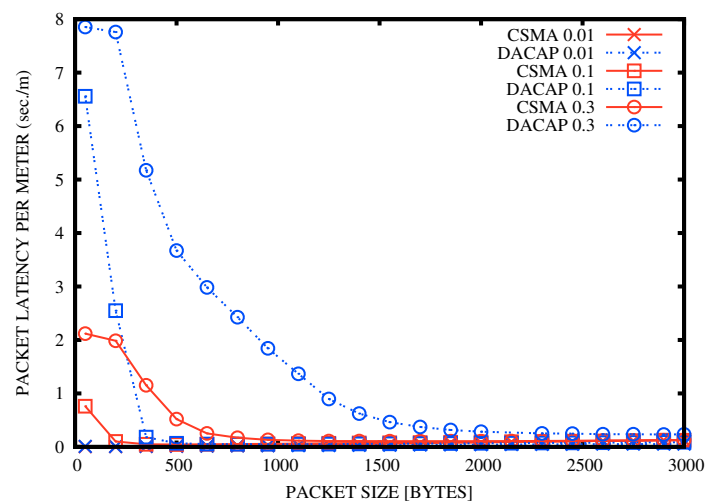

(b) $R=2000 \mathrm{bps}$

Figure 6. End-to-end latency per meter for $\mathrm{BER}=10^{-4}$.

of packets correctly delivered to the sink are generated by nodes close to it, while packets generated farther away are lost and do not reach the final destination (i.e. there is unfairness).

\section{Energy per bit}

Figures 8 and 9 show the energy consumption per bit per node. To investigate the average energy spent for each bit correctly delivered to the sink we consider the energy spent in transmitting, receiving and listening 


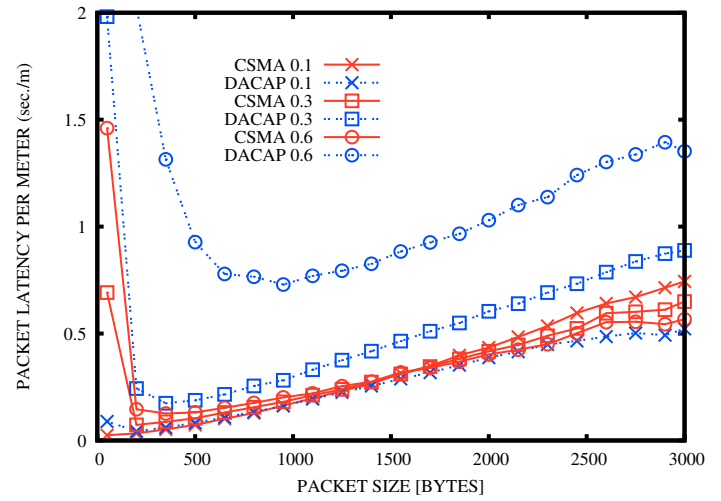

(a) $R=200 \mathrm{bps}$

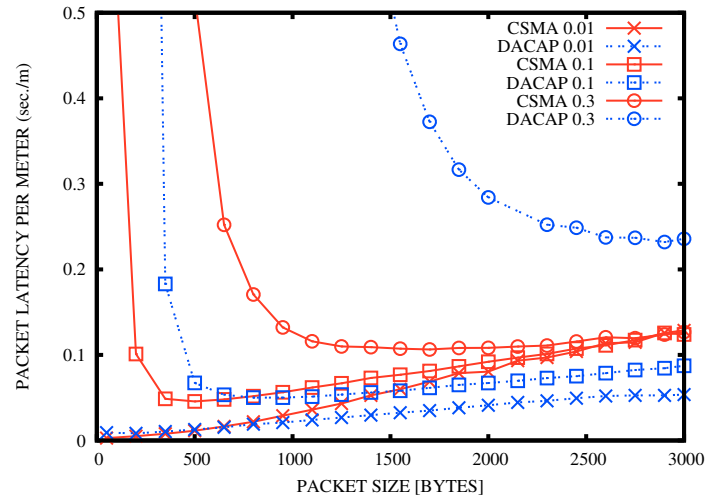

(b) $R=2000 \mathrm{bps}$

Figure 7. Zoom into the end-to-end latency per meter for $\mathrm{BER}=10^{-4}$.

to the channel. Nodes are always on, regardless of the traffic load and the packet size. The higher part of energy they spend is thus due to listening to the channel. This explains the performance improvement at medium traffic: The energy consumed for idle listening is offset by the higher number of delivered bits. When congestion builds up, packet delivery ratio decreases, increasing the energy consumption per bit.

Figure 8 (a) concerns scenarios with low BER $\left(10^{-6}\right)$ and nodes transmitting at 200bps. When the traffic is low nodes spend approximately the same energy, basically delivering all the bits and all being on for about the same time. We observe that when $\bar{\lambda} \geq 0.3$ both protocols experience the highest energy consumption per bit for short packets which imply more interference, more collisions and more re-transmissions. As the packet size increases, the energy consumed decreases.

A similar trend is observed in Figure $8(\mathrm{~b})$, for the same BER but higher bit rate (2000bps). Even though the throughput efficiency is lower than in the scenario with lower bit rate, more bits are correctly delivered to the sink. As a consequence, a much lower average energy per bit is required. Here, as before, we can see that using short packets, nodes consume more energy. For greater payloads, however, the energy per bit decreases. Differences in traffic patterns are justified by the corresponding number of bits delivered correctly, which agrees with what observed for throughput efficiency. Very noticeable is the case of very low traffic, for which we have a $100 \%$ efficiency. As expected, in this case, the average energy per bit is constant.

CSMA consumes about $10 \%$ to $16 \%$ more energy on average than DACAP for low/medium traffic, because of the higher number of re-transmissions. However, delivering more bits to the sink it pays less energy per bit.

The results for BER $=10^{-4}$ are shown in Figure 9.
Many of the considerations made for the lower BER apply here as well. For both protocols and bit rates, because of the lower number of bits correctly delivered to the sink due to the higher probability of error, the energy spent to deliver those bits increases with the packet size.

It is interesting to notice that while throughput efficiency and energy pet bit consumption are both strongly dependent on the number of bits correctly delivered to the sink thus showing best performance for similar packet sizes, packet latency shows a different behavior, especially when the transmission delay is longer (200bps). In this case, longer packets result in longer transmission delays.

\section{CONCLUSIONS}

We have investigated the impact of packet size on the performance of a multi-hop underwater acoustic network under two MAC protocols, CSMA and DACAP, whose simplicity makes them appealing candidates for practical system implementations. Simulation results show that the packet size is a fundamental parameter whose proper selection has a significant impact on throughput, latency, and energy consumption. They also quantify performance sensitivity to the choice of the packet size for the different protocols (CSMA and DACAP), emphasizing that a poorly informed selection of the packet size can result in a high cost to performance. We observed that the optimal packet size strongly depends on the protocol characteristics, on the BER, on the traffic load and on the data rate. Network designers should select the proper packet size based on the particular application requirements.

Future research will focus on packet size selection in networks with power control, different routing and multiple-packet acknowledgment schemes. 


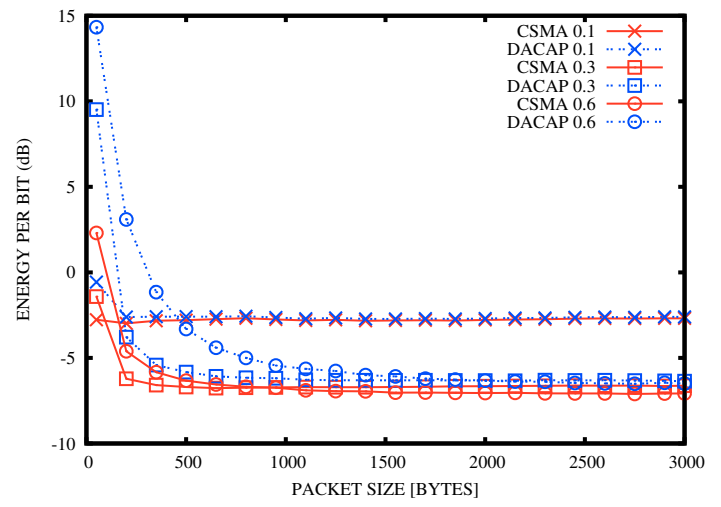

(a) $R=200 \mathrm{bps}$

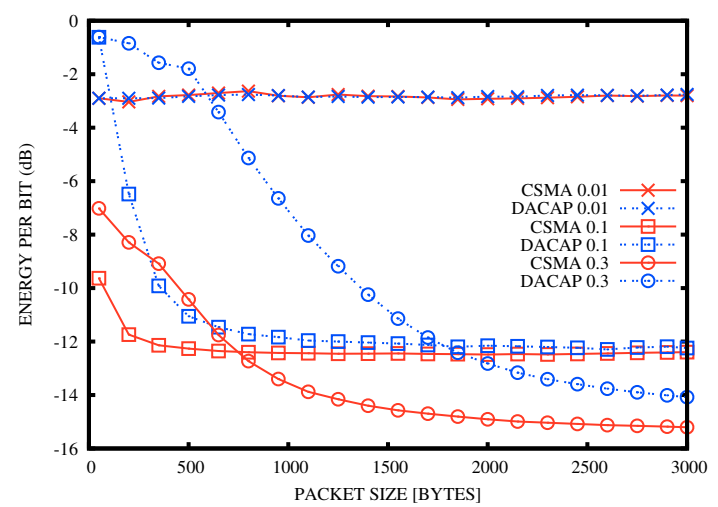

(b) $R=2000 \mathrm{bps}$

Figure 8. Energy per bit for BER $=10^{-6}$.

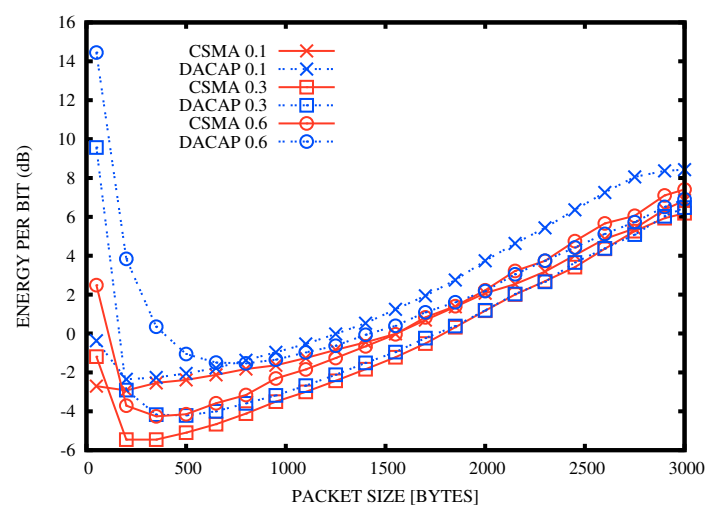

(a) $R=200 \mathrm{bps}$

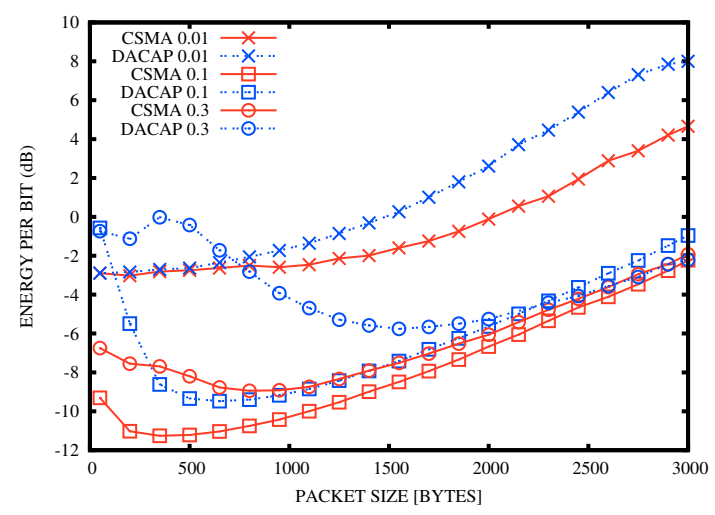

(b) $R=2000 \mathrm{bps}$

Figure 9. Energy per bit for BER $=10^{-4}$.

\section{ACKNOWLEDGMENTS}

This work was supported in part by the EU FP 7 STREP project CLAM "CoLlAborative EMbedded Networks for Submarine Surveillance " and by the NSF grants 0946610 and 0831728 .

\section{REFERENCES}

[1] I. F. Akyildiz, D. Pompili, and T. Melodia, "State of the art in protocol research for underwater acoustic sensor networks," SIGMOBILE Mobile Computing Communication Review, vol. 11, no. 4, pp. 11-22, October 2007.

[2] J. Heidemann, W. Ye, J. Willis, A. A. Syed, and Y. Li, "Research challenges and applications for underwater sensor networking," in Proceedings of the IEEE Wireless Communications and Networking Conference, WCNC 2006, Las Vegas, NV, April 3-6 2006, pp. 229-235.

[3] M. Chitre, S. Shahabudeen, and M. Stojanovic, "Underwater acoustic communications and networking: Recent advances and future challenges," Marine Technology Society Journal, vol. 42, no. 1, pp. 103-116, Spring 2008.

[4] N. Chirdchoo, W.-S. Soh, and K. C. Chua, "ALOHA-based MAC protocols with collision avoidance for underwater acoustic networks," in Proceedings of IEEE INFOCOM 2007, the 26th IEEE International Conference on Computer Communications, Anchorage, Alaska, USA, May 6-12 2007.
[5] X. Guo, M. Frater, and M. Ryan, "An adaptive propagation-delaytolerant MAC protocol for underwater acoustic sensor networks," in OCEANS 2007 - Europe, Aberdeen, Scotland, June 18-21 2007.

[6] J. M. Jornet, M. Stojanovic, and M. Zorzi, "Focused beam routing protocol for underwater acoustic networks," in WuWNeT '08: Proceedings of the third ACM international workshop on Wireless network testbeds, experimental evaluation and characterization, San Francisco, California, USA, September 15 2008, pp. 75-82.

[7] R. Nitzel, C. Benton, S. Chappell, and D. Blidberg, "Exploiting dynamic source routing to enable undersea networking over an ad-hoc topology," in Proceedings of the International Symposium on Underwater Technology, April 2002.

[8] B. Peleato and M. Stojanovic, "Distance aware collision avoidance protocol for ad-hoc underwater acoustic sensor networks." IEEE Communications Letters, vol. 11, no. 12, pp. 1025-1027, December 2007.

[9] C. Petrioli, R. Petroccia, and M. Stojanovic, "A comparative performance evaluation of MAC protocols for underwater sensor networks." in Proceedings of MTS/IEEE OCEANS 2008, Quebec City, Quebec, Canada, September 15-18 2008.

[10] D. Pompili, T. Melodia, and I. F. Akyildiz, "A resilient routing algorithm for long-term applications in underwater sensor networks." in Proceedings of IFIP Annual Mediterranean Ad Hoc Networking Workshop (Med-Hoc-Net), June 2006.

[11] A. Syed, W. Ye, and J. Heidemann, "Understanding spatiotemporal uncertainty in medium access with ALOHA protocols," in Proceedings of the Second ACM International Workshop on 
UnderWater Networks (WUWNet), September 2007, Montréal, Quebec, Canada, September 14 2007, pp. 41-48.

[12] - "Comparison and evaluation of the T-Lohi MAC for underwater acoustic sensor networks," IEEE Journal on Selected Areas in Communications, vol. 26, no. 9, pp. 1731-1743, December 2008.

[13] L. T. Tracy and S. Roy, "A reservation MAC protocol for adhoc underwater acoustic sensor networks," in WuWNeT '08: Proceedings of the third ACM international workshop on Wireless network testbeds, experimental evaluation and characterization, San Francisco, California, USA, September 15 2008, pp. 95-98.

[14] M. Zorzi, P. Casari, N. Baldo, and A. Harris, "Energy-efficient routing schemes for underwater acoustic networks," Selected Areas in Communications, IEEE Journal on, vol. 26, no. 9, pp. 1754-1766, December 2008.

[15] M. Stojanovic, "Optimization of a data link protocol for an underwater acoustic channel," in Proceedings of IEEE OCEANS 2005 - Europe, Brest, France, June 20-23 2005.

[16] A. S. Tanenbaum, Computer Networks, 4th ed. Upper Saddle River, NJ: Prentice Hall PTR, 2003.

[17] H.-H. Ng, W.-S. Soh, and M. Motani, "MACA-U: A media access protocol for underwater acoustic networks," in Proceedings of IEEE Globecom 2008, New Orleans, LA, November 30December 4 2008, pp. 1-5.

[18] The VINT Project, The ns Manual. http://www.isi.edu/nsnam/ns/, 2002.

[19] M. Stojanovic, "On the relationship between capacity and distance in an underwater acoustic communication channel," SIGMOBILE Mob. Comput. Commun. Rev., vol. 11, no. 4, pp. 34-43, 2007. 